\title{
Miranda
}

Revue pluridisciplinaire du monde anglophone /

Multidisciplinary peer-reviewed journal on the English-

speaking world

$12 \mid 2016$

Mapping gender. Old images ; new figures

\section{Notre top 9 des films anglophones de 2015}

\section{David Roche et Vincent Souladié}

\section{OpenEdition}

\section{Journals}

Édition électronique

URL : http://journals.openedition.org/miranda/8758

DOI : $10.4000 /$ miranda.8758

ISSN : 2108-6559

Éditeur

Université Toulouse - Jean Jaurès

\section{Référence électronique}

David Roche et Vincent Souladié, « Notre top 9 des films anglophones de 2015 », Miranda [En ligne],

12 | 2016, mis en ligne le 03 mars 2016, consulté le 16 février 2021. URL : http://

journals.openedition.org/miranda/8758; DOI : https://doi.org/10.4000/miranda.8758

Ce document a été généré automatiquement le 16 février 2021.

\section{c) (i)}

Miranda is licensed under a Creative Commons Attribution-NonCommercial-NoDerivatives 4.0 International License. 


\title{
Notre top 9 des films anglophones de 2015
}

\author{
David Roche et Vincent Souladié
}

1 Il y a eu beaucoup de bons films au cours de cette année 2015, mais peu de grands films. L'an dernier, certains des longs-métrages présentés ci-dessous n'auraient sans doute pas figuré dans notre liste.

2 Quelques cinéastes de renom ont réalisé des films tout à fait honorables : avec Bridge of Spies (Le Pont des espions, USA/Allemagne), Steven Spielberg prolonge les questionnements juridico-éthiques de Saving Private Ryan (Il faut sauver le soldat Ryan, 1998) et de Lincoln (2012) à travers une représentation de la Guerre Froide qui rejette tout manichéisme, malheureusement desservie par l'acharnement du cinéaste à intégrer l'histoire politique à l'histoire familiale; Todd Haynes continue de revisiter le mélodrame hollywoodien classique dans Carol (UK/USA), une histoire d'amour lesbien dont l'intégration contextuelle très convaincante est servie par une mise en scène et un jeu d'actrices à la fois sobre et somptueux; Inherent Vice (USA) de Paul Thomas Anderson transpose à l'écran de manière assez convaincante le roman réputé inadaptable de Thomas Pynchon, mais la version filmique de ce polar postmoderne ressemble finalement à un mélange entre The Big Lebowsky (Coen, 1998) et A Scanner Darkly (Linklater, 2006); Trainwreck (USA), marque la première incursion à Hollywood d'Amy Schumer, égérie trash du stand-up américain, sous les auspices avisés d'un Judd Apatow de plus en plus précis dans l'écriture comique de sa mise en scène, mais toujours aussi fidèle à son ton moralisateur ; The Visit (USA) de M. Night Shyamalan s'approprie intelligemment les lois esthétiques du found footage sans se départir de son style propre pour mettre en scène des personnages de jeunes adolescents très convaincants et très bien joués; et bien sûr Star Wars : Episode VII - The Force Awakens (Le Réveil de la Force, USA, J.J. Abrams) réussit le pari de plaire aussi bien aux fans de la trilogie originale (1977-1983) qu'aux générations actuelles, et pallie en même temps les manques de diversité des premiers films tout en incorporant un travail astucieux et pertinent sur la filiation et le masque. 
3 À cette liste peuvent s'ajouter quelques films de cinéastes prometteurs : Beasts of No Nation (USA, Cary Joji Fukunaga), production Netflix non sortie en salle, adapte un roman de l'écrivain américain d'origine nigérienne Uzodinma Iweala; porté par la performance d'Idris Elba, l'un des plus grands acteurs anglophones contemporains, bien servi par la mise en scène de Cary Joji Fukunaga, réalisateur de l'excellente première saison de True Detective (HBO, 2014), Beasts of No Nation harmonise la violence réaliste et l'onirisme à la Peter Pan pour nous faire découvrir l'histoire des guerres intestines nigériennes à travers le regard d'un enfant-soldat, interprété avec sensibilité par Abraham Attah; The Diary of a Teenage Girl (USA, Marielle Heller), premier film très réussi, film coming of age sans jugement, merveilleusement interprété par Bel Powley; The Duke of Burgundy (UK/Hongrie, Peter Strickland) met à jour le cinéma érotique des années 1970 avec un style visuel et sonore très léché (musique de Cat's Eyes), pour illustrer une histoire d'amour lesbien au beau renversement tragique entre Chiara D'Anna et Kata Bartsch ; Ex Machina (UK, Alex Garland), film de science fiction au récit Dickien assez convenu mais avec quelques scènes d'anthologie, dont une scène de danse post-humaine éblouissante ; 45 Years ( 45 ans, UK), troisième long métrage du cinéaste et scénariste Andrew Haigh, où un couple âgé, magnifiquement interprété par Tom Courtenay et Charlotte Rampling, voit son histoire subitement remise en question par l'intrusion du passé; Me and Earl and the Dying Girl (USA, Alfonso Gomez-Rejon), grand prix du jury à Sundance et deuxième long métrage très prometteur du jeune cinéaste latino américain connu pour ses épisodes de American Horror Story (FX, 2011-), teen flick qui aborde avec légèreté un sujet grave dans un style à la manière de Wes Anderson ; enfin, Tangerine (USA, Sean Baker), indie drôle et poignant avec des actrices/ personnages transgenres, filmé sur iPhone.

42015 a aussi connu son lot de déceptions : Joy (USA) de David O. Russell ne retrouve pas l'énergie d'American Hustle (American Bluff, USA, 2013); avec Knight of Cups (USA), Terrence Malick continue son projet ambitieux d'un cinéma de la mémoire et de la subjectivité, avec un personnage qui n'est pas sans rappeler ceux de Bret Easton Ellis, mais le résultat s'avère finalement assez confus et ennuyeux ; enfin, après le magistral Skyfall (UK/USA, 2012), Sam Mendes, tente à nouveau de raviver les James Bond des premiers temps avec Spectre (UK/USA), mais semble avoir joué tous ses atouts dans le film précédent.

5 On l'aura compris, la liste qui suit comprend surtout de bons films, mais aussi quelques grands films. Au lecteur de les deviner!

\section{The Big Short (USA, Adam McKay)}

6 Adam McKay a beau avoir réalisé les plus grandes réussites de la comédie américaine contemporaine (The Legend of Ron Burgundy, Step Brothers), sa crédibilité de cinéaste semblait se jouer, à lire certaines critiques, sur sa capacité à basculer dans le registre, inédit dans sa filmographie, du docu-drama sérieux. Or, la réussite de The Big Short repose justement sur le savoir faire de McKay pour conduire un récit aussi complexe avec l'énergie qui caractérise ses comédies, son goût pour la caricature des médiocres et la capacité avec laquelle certaines de ses meilleures scènes travaillent jusqu'à la syncope le mariage du grotesque puéril et du malaise. The Big Short parvient également à équilibrer un riche casting multi-protagonistes dans lequel chacune des stars à l'affiche donne à son interprétation une conviction qui évite le piège attendu du 
numéro de cabotinage (Steve Carell, Ryan Gosling, Christian Bale, Brad Pitt). Le financier zélé et irrascible ou l'investisseur autiste respectivement campés par Carell et Bale sont de nature à demeurer parmi leurs rôles les plus mémorables (mention spéciale pour la première apparition de Bale avec ce gros plan qui dure). Loin des réflexes mélodramatiques en vigueur dans ce genre de reconstitution, The Big Short distance, de notre point de vue, Spotlight (USA, Tom McCarthy, 2015) en terme de prise de risque esthétique. Partant d'une démarche identique, réunir un casting de vedettes (Michael Keaton, Rachel McAdams, Mark Ruffalo, Liev Schreiber et John Slattery) dans un film visant à dénoncer un scandale public, ce dernier, efficace, s'arme d'une gravité constante sans parvenir à dépasser sa facture de téléfilm de luxe. Dans The Big Short, au contraire, l'ambition pédagogique du récit, expliquer les tenants et les aboutissants de la crise des subprimes, confère au film une éthique qu'il prend en charge avec un humour et une vitalité propre aux moyens du cinéma, la qualité du montage et de la bande-son notamment. Cette démonstration de force traite son sujet avec une ludique férocité qu'Oliver Stone n'avait pas su retrouver lorsqu'il avait abordé précédemment le sujet (Wall Street: Money Never Sleeps, 2010). L'indignation critique n'est pas ici minorée par le rythme euphorique des situations, preuve en est l'absence de second degré cynique, de facilité manichéenne, ainsi que l'émotion distribuée par touches discrètes à l'égard des démunis laissés au ban de l'histoire par les malversations des personnages.

\section{Blackhat (Hacker, USA, Michael Mann)}

Blackhat représente à ce jour le plus grave échec commercial de la carrière de Michael Mann, symptôme d'un désaveu public déjà annoncé par la tiède réception de Miami Vice (2006) et de Public Enemies (2009). Son œuvre continue pourtant à attiser les débats. Pour une niche de fidèles aficionados Mann reste un auteur expérimental incompris du grand public, pour d'autres il est un auteur en disgrâce replié sans inspiration sur un genre unique qu'il auréole d'un maniérisme vain et répétitif. Blackhat est à ce titre délicat à appréhender en ce qu'il se donne a priori comme un œuvre " mineure » (un casting peu étoilé, un scénario de série B dont il n'est même pas l'auteur, un polar urbain de plus) et reconduit une large somme de motifs et de thématiques brassées depuis ses premiers films sans que se détermine a priori un autre usage celui de leur propre exposition, comme si le cinéaste, entre deux nouvelles tentatives d'opus magnum, voulait encore et seulement se rappeler à la mémoire. L'exercice est d'autant plus délicat que les décors du film conduisent le cinéaste à rivaliser ici avec ses propres légataires esthétiques, plus particulièrement Johnny To son héritier revendiqué. Il n'en reste pas moins que lorsque Michael Mann révise ses gammes il parvient encore, à défaut d'inventer, à singulariser des gestes arrachés à la fois à la banalité de la réalité et à la banalité du genre. Sans doute est-ce là l'une de ses lignes de force les moins reconnues, la finesse du regard posé sur le monde et le sens du détail grâce auquel ses saillies visuelles s'archivent dans notre mémoire presque à notre insu. Cette histoire plutôt grotesque de terrorisme informatique ponctuée d'inutiles plages de violence séduit alors par la concentration et l'application que met le cinéaste à filmer des mains glisser sur des claviers, la mise en scène substituant à cette passivité corporelle l'élan d'une urgence vitale et d'un corps à corps par machine interposée. En extériorisant de la même façon les arcanes de la logistique informatique à même le décor urbain (les 
piliers de béton apparentés à des barrettes de mémoire), Michael Mann trouve matière à relancer son obsession pour la réversibilité entre extérieur et intérieur.

\section{The Hateful Eight (Les Huit Salopards, USA, Quentin Tarantino)}

C'est peut-être le film le moins surprenant de Tarantino à ce jour, et donc forcément une déception à ce titre. Western à huis clos, La Chevauchée fantastique de John Ford qui rencontre Dix petits nègres d'Agatha Christie, film et roman sortis tout deux en 1939, Les Huit salopards se présente comme une œuvre somme, synthèse de son premier film Reservoir Dogs (1991) et de son avant-dernier Django Unchained (2012). On retrouve plusieurs hommes enfermés dans une même pièce avec une taupe dont on ignore l'identité. La réussite de The Hateful Eight se situe peut-être moins au niveau de son récit qu'à la manière dont le film articule parfaitement son ambition politique à son ambition plastique. Le recours au $70 \mathrm{~mm}$ n'est pas juste une lubie du cinéaste, il a bel et bien une fonction esthétique : bâtir un film sur l'opposition entre la splendeur du paysage westernien et la noirceur du huis clos. C'est à ce niveau que la référence à $L a$ Chose (1982) de Carpenter, à travers le casting de Kurt Russell et l'emploi de la musique composée par Morricone (mais laissée de côté par Carpenter), prend tout son sens. Comme Corbucci et Leone, deux de ses mentors, Tarantino fait appel à l'imagerie mythique du Western classique pour déconstruire la version de l'histoire qu'il promeut. Dans une station en montagne, on reprend l'histoire américaine en rejouant la célèbre scène finale de Naissance d'une nation (1915) de Griffith, scène déjà référencée dans Django Unchained. Nord et Sud s'opposent autour des questions raciales, le bourreau britannique jouant le rôle du médiateur afin de sécuriser l'espace en le divisant. Au milieu du film vient alors la première scène de violence, quand le chasseur de primes afro-américain, Warren, provoque un ancien général sudiste en lui racontant comment il a violé puis tué son fils, le tout dans le but de venger ses frères exécutés par ce même général. C'est alors en flashback que cette souillure vengeresse nous est montrée au cœur même du paysage westernien. La suite du récit repose alors sur la question suivante : l'avarice et le racisme vont-ils empêcher la réconciliation entre nord et sud, entre noir et blanc? Une réconciliation qui vient à refuser la figure de l'innocence brandie dans le film de Griffith, diabolisée dans celui de Tarantino.

\section{Inside Out (Vice-versa, USA, Peter Docter et Ronnie Del Carmen)}

Depuis son rachat par l'empire Disney en 2006, il est devenu commun pour les critiques d'annoncer l'inexorable déclin artistique de Pixar. Cet argument secundum quid désole car il est simplement déduit par l'antipathie manichéenne à l'égard de l'épouvantail capitaliste que représente Disney. Par esprit de contradiction, on pourrait rétorquer à cette rengaine que l'apport de Pixar promet d'entraîner à long terme une revalorisation esthétique des œuvres Disney. En tout cas, si le mariage des deux compagnies a effectivement poussé l'orientation de l'œuvre de Lasseter vers des longs-métrages rentables auprès du jeune public (Cars, 2006), il est possible de croire en une perpétuation de la qualité historique des films Pixar sur la foi de sa production 
régulière de longs-métrages exemplaires, mais fallacieusement reçues comme des exceptions à la déroute artistique annoncée. Sans doute moins connu que Brad Bird ou Andrew Stanton, Pete Docter est présent dans l'écurie Pixar depuis ses débuts et a bénéficié de son principe de promotion interne jusqu'à écrire et réaliser avec Up (2009), l'une des plus belles réussites de la compagnie. Inside Out le fait bénéficier d'une même liberté créatrice et s'inscrit avec une parfaite cohérence dans la ligne par ailleurs tracée par Monsters, Inc. (2001), WALL-E (2008) ou Toy Story 3 (2010), celle des œuvres alliant le plaisir poétique des récits enfantins à la profondeur et à la gravité dramatique. Pete Docter impressionne en l'occurrence par son refus d'infantiliser le public et de simplifier les enjeux psychanalytiques de son récit, autant que par la facilité synthétique avec laquelle il conceptualise le labyrinthe mental du jeune personnage. Les différentes couches de sens du régime figuratif plongent ainsi le spectateur dans un univers à la fois spatial et mémoriel, chimérique et concret. Inside Out fait ainsi subir à l'action les incohérences d'un désordre démiurge insaisissable (le déchaînement incohérent des émotions pubères) tout en traduisant le récit d'initiation en une odyssée dépressive mais salvatrice vers l'oubli, l'effondrement de l'innocence comme condition d'apprentissage et de maturité. Comme Monsters, Inc. et Toy Story 3 avant lui, Inside Out refuse la complaisance du syndrome de Peter Pan, ce qui ne l'empêche pas de revisiter et de retravailler l'héritage Disney pour en assurer la continuité (Dumbo, Pinnochio, Alice aux Pays de Merveilles). Inside Out ajoute par ailleurs à ses prédécesseurs un point de vue assez nouveau chez Pixar, doublement représenté ici par le couple parental et par les émotions incarnées de la jeune Riley, en particulier la chef de file Joy : celui des parents qui observent avec autant de mélancolie que de fierté l'inexorable évolution de leur enfant et doivent apprendre, à l'instar de l'ami imaginaire Bing Bong, à faire le deuil de l'idée qu'ils seront toujours là pour le protéger.

\section{It Follows (USA, David Robert Mitchell)}

10 Lauréat du Grand Prix du Festival du Film Fantastique de Gérardmer 2015, le second long métrage de Robert Mitchell est sans aucun doute le film d'horreur indépendant américain le plus réussi depuis Le Projet Blair Witch (Myrick et Sánchez, 1999) et Saw (Wan, 2004). Contrairement à ces deux films qui ont largement participé à la création et à la popularité de deux sous-genres, le found footage et le torture-porn, la réussite de It Follows réside, en premier lieu, dans un retour aux fondamentaux: à savoir à une esthétique tributaire de Val Lewton et Jacques Tourneur (la scène de la piscine renvoie à Cat People [La Féline, 1942]), de George A. Romero (l'effet de sidération produit par un monstre lent et maladroit) et surtout du John Carpenter de Halloween (1978) et Fog (1980) (pour la composition et la musique minimaliste au synthé). À l'ère des zombies qui courent et des spectres qui font sursauter, It Follows est un film qui prend son temps... et on lui en sait gré : les apparitions dans le parking, le lycée et la maison sont terrifiantes. L'efficacité plastique impressionne, notamment l'affection de Mitchell pour les plans larges, longs et lents, lesquels étaient déjà présents dans son premier film, The Myth of the American Sleepover (2010), tout comme l'insistance de la caméra sur le regard comme expression et vecteur du désir adolescent. Après Lost River (Gosling, 2014) et surtout l'envoutant Only Lovers Left Alive (Jarmusch, 2013), It Follows offre également une vision gothique de la ville de Detroit, capitale de l'automobile américaine devenue ville cimetière dans laquelle les personnages errent, justement, en voiture. Avec ses adolescents dépeints sans aucune condescendance, It Follows poursuit 
la réflexion sur les relations amoureuses de la jeunesse américaine contemporaine entamée dans The Myth of the American Sleepover, tout en revisitant également les soustextes des slashers des années 1980, avec une créature changeante (comme le ça de Stephen King) qui n'incarne pas tant la figure du père castrateur que celle de la contamination sexuelle. On peut suivre Tarantino quand il dit de It Follows qu'il s'agit d'un film terriblement frustrant parce qu'il manque tout juste d'être un " film génial ${ }^{1}$ ». Toujours est-il qu'avec un budget de $\$ 2$ million, Mitchell réussit là où la majorité des films d'horreur américains contemporains dotés de budget souvent plus conséquents échouent : à proposer une œuvre terrifiante et d'une intelligence rare.

\section{The Lobster (Irlande/UK/Grèce/France/Pays-Bas/USA, Yorgos Lanthimos)}

11 Production internationale s'il en est, Lanthimos prouve, avec son cinquième long métrage, que le cinéma transnational ou pan-européen n'est pas forcément un cinéma du compromis, comme l'avait montré Lars Von Trier avant lui. Dans la droite lignée de son troisième long métrage Canine (Kynodontas, Grèce, 2009), remarqué dans la catégorie "Un Certain Regard " à Cannes, Yorgos propose un univers étrange, au carrefour de diverses influences cinématographiques (Herzog et Tarkovsky) et surtout littéraires (Kafka, et les récits dysptopiques de Barjavel, Huxley, Orwell et Bradbury). La structure du récit est elle-même fondée sur ces influences littéraires : une première partie au postulat kafkaïen - les personnages célibataires intègrent un hôtel dans lequel ils doivent trouver un partenaire qui leur ressemble, au risque d'être transformés en l'animal de leur choix - et une seconde plus classique basée, en apparence, sur l'opposition entre nature et culture. Si le cinéma dystopique abonde et compte un grand nombre de réussite - Metropolis (1927), Alphaville (1965), Farenheit 451 (1966), Soleil Vert (1973), Stalker (1979), Blade Runner (1982), Brazil (1985), Gattaca (1997) -, les récits de Kafka, voire leur esprit même, sont réputés difficilement transposables au cinéma, et cela malgré toute l'estime que nous avons pour Le Procès (1962) de Welles. Or, The Lobster mêle habilement pathos, absurde, humour - on pense au poney blond qui apparaît à deux reprises dans le film - dans un récit qui s'interroge sur l'articulation entre la loi et les relations humaines : d'un côté, un régime qui impose le couple, de l'autre côté un contre-pouvoir qui le rejette tout aussi brutalement. Autrement dit, les discours et les pratiques normatifs véhiculés par pouvoir et contre-pouvoir, au-delà d'une opposition entre une société capitaliste dont l'hôtel est la synecdoque et une communauté faussement anarchiste et superficiellement associée à la nature, régissent, selon des modalités similaires, des situations qui relèvent du sensible et de la contingence. Même le couple qui se forme à la marge n'y échappe pas, ayant déjà intégré un discours auquel il adhère maintenant qu'il semble expliquer leur situation. La question que le film choisit de laisser ouverte, à travers une fin sans issue qui rappelle celle de Canine, n'est donc pas autant le degré d'amour qu'un partenaire est capable de manifester pour l'autre au nom du sacrifice, que, encore une fois comme dans Canine, le degré d'assujettissement de personnages qui finalement cherchent le refuge dans la structure sociale même qui les avait préalablement opprimés, les « jeux interdits " du désir étant finalement autorisés par la société «oppressive ». Il me semble que ce n'est donc pas uniquement au niveau de la photographie aux tons pâles 
que l'on retrouve l'influence de Tarkovsky, mais également dans son recours à l'allégorie pour traiter de grandes questions existentielles.

\section{Mad Max: Fury Road (Australie/USA, George Miller)}

12 Pour George Miller, parier sur le retour de Mad Max trente ans après le précédent opus ne pouvait se faire sans quelques gages de fidélité aux fondamentaux de la célèbre franchise australienne. Après trois épisodes plutôt différents les uns des autres, cet addendum à la saga ne creuse pas vraiment les possibilités dramatiques de cet univers mais reprend peu ou prou la trame narrative de Road Warrior, c'est-à-dire le film qui installa le personnage de Max et sa réalité post-apocalyptique au rang des figures iconiques du cinéma. Road Warrior fut celui qui donna lieu à une génération de succédanés, aussi bien aux États-Unis qu'en Europe, qui tout en pillant le film participèrent à accroître son aura. Peu surprenant pour réactiver la franchise que d'aller puiser à cette même source, au risque même de placer Fury Road dans l'ombre de son aîné. L'attente est peut-être même entachée d'une déception de principe à ne pas à ne pas voir Miller statuer de façon transparente sur la nature de son nouveau film, suite, remake ou reboot, comme si le cinéaste était paralysé par les repères imposants qu'il avait jadis inventé. Fort heureusement, cette contrariété est très vite balayée par l'énergie quasi nihiliste $\mathrm{du}$ film, lequel n'envisage en fait le recyclage narratif que comme la voie la plus immédiate vers son projet esthétique : l'expression pure de la mise en scène dégagée de tout excédent d'histoire et de faux prétextes dramatiques. CEuvrant en terrain conquis, Miller s'offre la liberté de réduire l'exposition de son univers à quelques traits pour proposer un idéal de cinéma de genre dans lequel les différents enjeux, les rapports de force entre les personnages et leur évolution, sont révélés dans l'action et articulés au montage en flux tendu sans recours à une surexplication verbale annexe. L'ensemble fonctionne comme un immense film d'animation à la Chuck Jones, bruyant et coloré, porté par une frénésie inépuisable qui se donne pour projet l'ivresse sensorielle permanente. L'enjeu rythmique se confond à l'enjeu narratif qui le justifie à tel point que Miller, ayant déjà épuisé toutes les cartouches de son histoire, se permet à mi-chemin un ironique sursaut réflexif qui n'a pas manqué de provoquer quelque irritation : arrivés au terme de leur quête après une longue et frénétique course-poursuite, et n'ayant découvert que vide et désolation en lieu et place du paradis terrestre promis, les personnages choisissent de faire demi-tour jusqu'à leur point de départ ! Cette logique rythmique ne résume pas à elle seule le film, puisque celui-ci s'offre comme prise de risque inattendue la mise en retrait de son personnage éponyme. L'interprétation de Tom Hardy vise d'abord à habiller son jeu de touches expressives pour conférer au rôle une humanité fragile, à l'opposé de l'icône impavide à laquelle Mel Gibson prêta jadis ses traits. Cette caractérisation bienvenue, à mettre au crédit du comédien, s'ajoute au sort que lui réserve l'action, à laquelle, justement, il ne participe quasiment pas. Max se voit en effet immobilisé par ses adversaires pendant que le contingent de figures féminines, d'abord présentées comme des victimes et des proies à sauver, s'accomplissent héroïquement. Miller destitue Mad Max de sa figure virile pour l'incrémenter d'un féminisme plus ou moins actuel qui peut renvoyer son western apocalyptique aussi bien à certains films d'exploitation des années soixante-dix (She-Devils on Wheels, Herschell Gordon Lewis, 1968, Unholy Rollers, Vernon Zimmerman, 1972) qu'au Westward the Women de William Wellman (1951). 


\section{Room (Irlande/Canada, Lenny Abrahamson)} confirmer son talent avec ce cinquième long métrage, vainqueur aux British Independent Film Awards et trois fois nommé aux Oscars (meilleur cinéaste, meilleur rôle féminin, meilleure adaptation). "Room» c'est, comme l'absence d'article en anglais l'indique, le monde dans lequel vivent Ma et son fils Jack. Cocon? prison? dystopie? L'univers extérieur ne pénètre dans cette chambre qu'à travers le velux, les personnages deux-dimensionnels de la télévision et old Nick, l'homme qui apporte des provisions la nuit et a des rapports avec Ma alors que Jack est couché. Abrahamson s'inscrit dans la droite filiation de Fallen Idol (Première désillusion, 1948), l'un des chefs d'œuvre de Carol Reed, afin de nous proposer habilement une alternance entre l'incompréhension de l'enfant sur son environnement et le vécu de la mère. La réussite $\mathrm{du}$ film tient avant tout à un récit dont les rebondissements n'ont rien de tours de passe-passe, mais reposent sur un travail sur le point de vue qui vise l'émotion plutôt que le suspense. On s'en doute, le mystère de la chambre n'est qu'une étape dans une aventure émotionnelle forte reposant sur une dialectique intérieur-extérieur (l'espace physique et psychique) qui évite véritablement toutes les facilités (sauf peut-être la musique un peu convenue): ni manichéisme ni misérabilisme ni célébration d'une résilience héroïque, juste la dure réalité du trauma. Le talent d'Abrahamson réside dans sa capacité à allier des tons difficilement compatibles, comme dans cette scène de poursuite à la fois contemplative et angoissante. Enfin, Room, c'est aussi la réussite de ses acteurs : Brie Larson et le jeune Jacob Tremblay.

\section{Sicario (USA, Dennis Villeneuve)}

Après le touchant Incendies (2010), le magistral Prisoners (2013) et l'atmosphérique Enemy (2013), on espérait que le septième film du cinéaste canadien Dennis Villeneuve soit celui de la consécration. Une fois la déception passée, on doit se rendre à l'évidence qu'il s'agit néanmoins d'une confirmation. Comme Prisoners, Sicario est le film antiaméricain par excellence aux niveaux politique et générique. L'officière du FBI Kate Macer (Emily Blunt) se laisse convaincre par sa hiérarchie que l'opération à laquelle elle accepte de participer repose sur une légitime justice ; le personnage de Matt Graver (Josh Brolin), toujours le sourire aux lèvres, nous promet aussi de l'enthousiasme dans les scènes d'action. Il n'en est rien. L'héroïne n'intègre jamais pleinement ni le récit ni le complot, et traverse le film comme témoin, comme double du spectateur, manipulé et choqué. À l'instar d'Incendies, Sicario est l'histoire d'une frontière, mais ici la révélation ne conduit pas à une réconciliation; elle ne fait que renforcer le sentiment d'impuissance et d'étouffement qui domine le film. Les plans d'ensemble en plongée de la frontière et la bande son annoncent la couleur : nous n'auront droit à aucun répit. Le récit s'engouffre dans les ténèbres, les tunnels sous la frontière n'offrent aucune échappatoire aux misérables, ils ne représentent qu'un prétexte pour le pouvoir de transgresser les lois géopolitiques et morales. La politique américaine est ici pointée du doigt dans toute son abjection: sa trouble coalition avec le mal contre lequel elle prétend lutter, et son éthique réduite à un seul précepte biblique, œil pour œil, dent pour dent. 


\section{NOTES}

1. Film: blogging the real. http://www.slashfilm.com/quentin-tarantino-it-follows/. Vu le 10 décembre 2015.

INDEX

Mots-clés : cinéma, Australie, Canada, Grande Bretagne, Grèce, Irlande, Etats-Unis

\section{AUTEURS}

\section{DAVID ROCHE}

Professeur d'études filmiques

mudrock@neuf.fr

\section{VINCENT SOULADIÉ}

Docteur en études cinématographiques

vsouladi@univ-tlse2.fr 